\title{
Pruning Neural Networks with Distribution Estimation Algorithms
}

\author{
E. Cantu-Paz
}

This article was submitted to: Genetic and Evolutionary Computation Conference, Chicago, IL, USA 07/12/2003 - 07/16/2003

\section{January 15, 2003}

\section{U.S. Department of Energy}

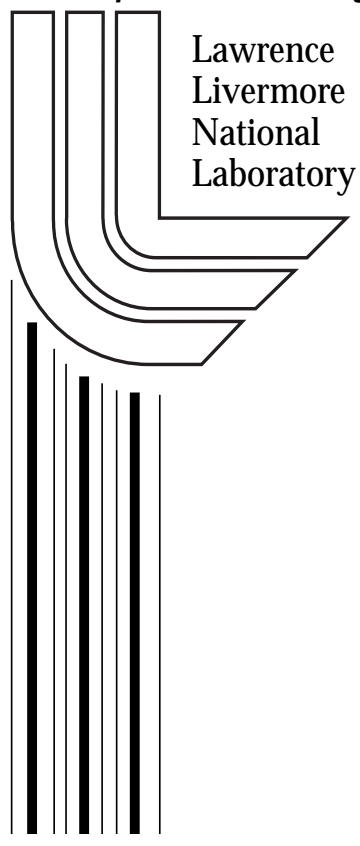




\section{DISCLAIMER}

This document was prepared as an account of work sponsored by an agency of the United States Government. Neither the United States Government nor the University of California nor any of their employees, makes any warranty, express or implied, or assumes any legal liability or responsibility for the accuracy, completeness, or usefulness of any information, apparatus, product, or process disclosed, or represents that its use would not infringe privately owned rights. Reference herein to any specific commercial product, process, or service by trade name, trademark, manufacturer, or otherwise, does not necessarily constitute or imply its endorsement, recommendation, or favoring by the United States Government or the University of California. The views and opinions of authors expressed herein do not necessarily state or reflect those of the United States Government or the University of California, and shall not be used for advertising or product endorsement purposes.

This is a preprint of a paper intended for publication in a journal or proceedings. Since changes may be made before publication, this preprint is made available with the understanding that it will not be cited or reproduced without the permission of the author.

This report has been reproduced directly from the best available copy.

Available electronically at http://www.doc.gov/bridge

Available for a processing fee to U.S. Department of Energy

And its contractors in paper from

U.S. Department of Energy

Office of Scientific and Technical Information

P.O. Box 62

Oak Ridge, TN 37831-0062

Telephone: (865) 576-8401

Facsimile: (865) 576-5728

E-mail: reports@adonis.osti.gov

Available for the sale to the public from

U.S. Department of Commerce

National Technical Information Service

5285 Port Royal Road

Springfield, VA 22161

Telephone: (800) 553-6847

Facsimile: (703) 605-6900

E-mail: orders@ntis.fedworld.gov

Online ordering: http://www.ntis.gov/ordering.htm

\section{OR}

Lawrence Livermore National Laboratory

Technical Information Department's Digital Library

http://www.llnl.gov/tid/Library.html 


\title{
Pruning Neural Networks with Distribution Estimation Algorithms
}

\author{
Erick Cantú-Paz \\ Center for Applied Scientific Computing \\ Lawrence Livermore National Laboratory \\ Livermore, CA 94551 \\ cantupaz@llnl.gov
}

\begin{abstract}
This paper describes the application of four evolutionary algorithms to the pruning of neural networks used in classification problems. Besides of a simple genetic algorithm (GA), the paper considers three distribution estimation algorithms (DEAs): a compact GA, an extended compact GA, and the Bayesian Optimization Algorithm. The objective is to determine if the DEAs present advantages over the simple GA in terms of accuracy or speed in this problem. The experiments used a feedforward neural network trained with standard backpropagation and public-domain and artificial data sets. The pruned networks seemed to have better or equal accuracy than the original fully-connected networks. Only in a few cases, pruning resulted in less accurate networks. We found few differences in the accuracy of the networks pruned by the four EAs, but found important differences in the execution time. The results suggest that a simple GA with a small population might be the best algorithm for pruning networks on the data sets we tested.
\end{abstract}

\section{Introduction}

The success of neural networks (NNs) largely depends on their architecture, which is usually determined by a trial-and-error process. There are numerous combinations of neural networks and evolutionary algorithms (EAs) to optimize the network architecture to reach the highest possible classification accuracy [1, $2]$. In the present paper, we examine neural network pruning by four evolutionary algorithms to improve the generalization accuracy in classification problems.

We experimented with a simple genetic algorithm (sGA) and three distribution estimation algorithms (DEAs): a compact GA (cGA), an extended compact GA (ecGA), and the Bayesian Optimization Algorithm (BOA). Instead of the mutation and crossover operations of conventional GAs, DEAs use a statistical model of the individuals that survive selection to generate new individuals. Numerous experimental and theoretical results show that DEAs can solve hard problems reliably and efficiently [3-5].

The objective of this study is to determine if DEAs present advantages over simple GAs in terms of accuracy or speed when applied to neural network pruning. The networks used in the experiments were conventional feedforward per- 
ceptrons with one hidden layer and were trained with the backpropagation algorithm. The experiments used 13 public-domain and two artificial data sets.

Our target was to maximize the accuracy of classification. The experiments demonstrate that in most cases, pruned networks have at least as good accuracy as fully-connected networks. We found few significant differences in the accuracy of networks pruned by the four EAs, but found important differences in the execution time.

The next section presents background on neural network pruning, including some previous applications of EAs to pruning. Section 3 describes the algorithms, data sets, and the method used to compare the algorithms. The experimental results are presented in section 4 . Section 5 concludes this paper with a summary, the conclusions of this study, and a discussion of future research directions.

\section{Neural Network Pruning}

It is well known that a network that is too big for a particular classification task is more likely to overfit the training data and have poor performance on unseen examples (i.e., poor generalization) than a small network. Therefore, a heuristic to obtain good generalization is to use the smallest network that will learn to classify correctly the training data. However, the optimal network size is usually unknown and tedious experimentation becomes necessary to find a good network. An alternative to improve generalization is to train a network that is believed to be larger than necessary and prune the excess parts.

Numerous algorithms have been used to prune neural networks [6]. Pruning begins by training a fully-connected neural network. Most pruning methods delete a single weight at a time in a greedy fashion, which may result in suboptimal pruning. Additionally, many pruning methods fail to account for the interactions between multiple weights. This may be problematic if deleting one weight makes it appear as if another weight that should be pruned is important for the operation of the network. An algorithm that considers weight interactions and more than one weight at a time may have better chances of reducing the size of the network significantly without affecting the classification accuracy. For these reasons, GAs and DEAs seem promising for NN pruning.

Genetic algorithms have been used to prune networks and are reported to deliver good results [7-9]. Applying GAs to prune networks is straightforward: The chromosomes contain one bit for each weight, and the value of the bit determines whether the weight will be used in the final network. This simple binary encoding is used in the experiments in the present paper. Other more sophisticated methods of simultaneously training and pruning the networks were introduced by Schmidt and Stidsen [10].

Whitley [11] suggests to retrain the network for a few epochs after pruning the weights. We performed experiments to confirm this idea, but our experiments show only limited advantages of retraining. 
It is also possible to prune entire (input and hidden) nodes, but in the present paper we experiment only with the more common approach of pruning individual weights. We leave pruning units to future work.

\section{Methods}

This section describes the algorithms and the data sets used in this paper as well as the statistical method used to compare the algorithms.

\subsection{Algorithms}

The simple genetic algorithm in this study uses binary strings, binary (pairwise) tournament selection without replacement, uniform crossover, and bitwise point mutation. Simple GAs such as this have been used successfully in many applications. However, it has long been recognized that the problemindependent crossover operators used in simple GAs can disrupt groups of related variables and prevent the algorithm from reaching the global optimum, unless exponentially-sized populations are used. (Thierens [12] gives a good description of this problem).

One approach to identify and exploit the relationships among variables is to estimate the joint distribution of the individuals that survive selection and use this model to generate new individuals. The complexity of the models has increased over time as more sophisticated methods of building models from data and more powerful computers become available. Interested readers can consult the reviews by Pelikan et al. [13] and Larrañaga et al. [14].

The simplest model-building EA used in the experiments reported here is the compact GA [15]. This algorithm assumes that the variables (bits) that

represent the problem are independent, and therefore it models the population as a product of Bernoulli distributions. The compact GA receives its name from the compact way it represents the population: The cGA uses a vector $p$ of length equal to the problem's length, $l$. Each element of $p$ contains the probability that a sample will take the value 1 . If the Bernoulli trial is not successful the sample will be 0 . All positions of $p$ are initialized to 0.5 to simulate the usual uniform random initialization of simple GAs. New individuals are obtained by sampling consecutively from each position of $p$ and concatenating the values obtained. The probabilities vector is updated by comparing the fitness of two individuals obtained from it. For each $p_{k}, k=1, . ., l$, if the fittest individual has a 1 in the $k$-th position, $p_{k}$ is increased by $1 / n$, where $n$ is the size of the virtual population that the user wants to simulate. Likewise, if the fittest individual has a 0 in the $k$-th position, $p_{k}$ is decreased by $1 / n$. The cGA iterates until all positions in $p_{k}$ contain either zero or one.

PBIL [16] and the UMDA [17] are other examples of algorithms that use univariate models and operate on binary alphabets. They differ from the cGA in the method to update the probabilities vector. 
The extended compact GA [18] uses a product of marginal distributions on a partition of the variables. In this model, subsets of variables can be modeled jointly, and the subsets are considered independent of other subsets. Formally, the model is $P=\prod_{i=0}^{m} P_{i}$, where $m$ is the number of subsets in a partition of the variables and $P_{i}$ represents the distribution of the $i$-th subset. The distribution of a subset with $k$ members is stored in a table with $2^{k}-1$ entries. The challenge is to find a partition that models the population correctly. Harik [18] proposed a greedy search that initially supposes that all variables are independent. The model search tries to merge all pairs of subsets and chooses the merger that minimizes a complexity measure based on information theory. The search continues until no further subsets can be merged. In contrast to the cGA, the ecGA has an explicit population that is evaluated and subject to selection at each iteration of the algorithm. The algorithm builds the model considering only those solutions that survive selection. The population is initialized randomly, and new individuals are generated by sampling consecutively from the $m$ subset distributions.

The Bayesian Optimization Algorithm [3] models the selected individuals using a Bayesian network, which can represent dependence relations among an arbitrary number of variables. Independently, Etxeberria and Larrañaga [4] and Mühlenbein and Mahnig [5] introduced similar algorithms. The BOA uses a greedy search to optimize the Bayesian Dirichlet metric, a measure of how well the network represents the data (the BOA could use other metrics). The user specifies the maximum number of incoming edges to any node of the network. This number corresponds to the highest degree of interaction assumed among the variables of the problem. As the ecGA, the BOA builds the model considering only the solutions that survived selection. New individuals are generated by sampling from the Bayesian network.

The main difference between the ecGA and the BOA is the model that they use to represent the survivors. Figure 1 illustrates the different models used by the ecGA and the BOA. The ecGA cannot represent individual relationships among the variables in a subset.

The experiments used the $\mathrm{C}++^{+}$implementations of the ecGA [19] and the BOA version $1.0[20]$ that are distributed by their authors on the web (at http://www-illigal.ge.uiuc.edu). The ecGA code has a non-learning mode that emulates the cGA. The sGA and the neural network were developed in $\mathrm{C}++$. All programs were compiled with $\mathrm{g}++$ version 2.96 using -O2 optimizations. The experiments were executed on a single processor of a Linux (Red Had 7.2) workstation with dual $2.4 \mathrm{GHz}$ Intel Xeon processors and $512 \mathrm{Mb}$ of memory. The ecGA and the BOA codes were modified to use a Mersenne Twister random number generator, which was also used in the GA and the data partitioning.

The algorithms used populations with 1024 individuals and were initialized uniformly at random. The GA used uniform crossover with probability 1.0, and mutation with probability $1 / l$, where $l$ was the length of the chromosomes and corresponds to the total number of weights in the network. Promising solutions were selected with pairwise binary tournaments without replacement. The cGA, ecGA, and the BOA used the default parameters provided in their distributions: 


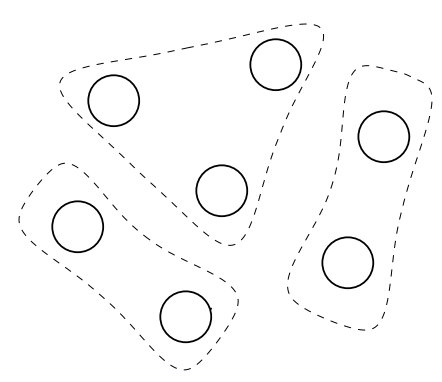

(a) ecGA

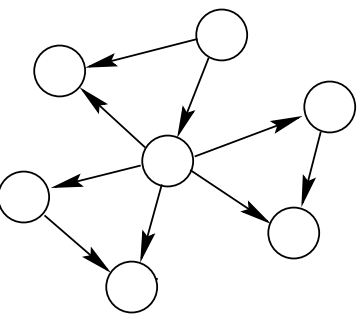

(b) BOA

Fig. 1. Representation of the models used in the ecGA and the BOA.

The cGA and ecGA used tournaments among 16 individuals, and the BOA used truncation selection with a threshold of $50 \%$. All algorithms were terminated after observing no improvement in the best individual over five consecutive generations, or until a limit of 50 generations was reached.

The network used in the experiments was a fully-connected perceptron with a single hidden layer. The hidden and output units compute their output as $f($ net $)=\tanh ($ net $)$, where net $=\sum_{i=1}^{d} x_{i} w_{i}+w_{0}$ is the net activation, the $x_{i}$ are inputs to the unit, $w_{i}$ are the connection weights and $w_{0}$ is a bias term. The weights were initialized uniformly at random in the interval $[-1,1]$. Before each EA run, a network was trained with simple backpropagation using a learning rate of 0.15 and a momentum term of 0.9. The sizes of the network and the number of training epochs varied for each data set and are specified in table 1.

For all the algorithms, the classification accuracy of the pruned network on the training data served as the fitness function. In cases where the pruned network is retrained with backpropagation, the algorithms exploit the Baldwin effect: The retrained pruned network is used to evaluate the fitness, but the retrained weights are not inherited. Note that the fitness measure does not bias the search explicitly toward networks with few weights.

\subsection{Data Sets}

The data sets used in the experiments are described in table 1 . The data sets are available in the UCI machine learning repository [21], except for Random21 and Redundant21, which are artificial data sets with 21 features each. The target concept of these two data sets is to define whether the first nine features are closer to $(0,0, \ldots, 0)$ or $(9,9, \ldots, 9)$ in Euclidean distance. The features were generated uniformly at random in the range [3,6]. All the features in Random21 are random, and the first, fifth, and ninth features are repeated four times each in 
Table 1. Description of the data sets used in the experiments. For each data set, the table shows the number of instances; the number of classes; the number of continuous and discrete features; the number of input, hidden, and output units; and the number of epochs of backpropagation used to train the networks.

\begin{tabular}{l|cc|cc|cccc}
\hline & & & \multicolumn{5}{|c|}{ Features } & \multicolumn{5}{c}{ Neural Network } \\
Domain & Cases Class & Cont. Disc. & Input & Output & Hidden Epochs \\
\hline Breast Cancer & 699 & 2 & 9 & - & 9 & 1 & 5 & 20 \\
Credit-Australian & 653 & 2 & 6 & 9 & 46 & 1 & 10 & 35 \\
Credit-German & 1000 & 2 & 7 & 13 & 62 & 1 & 10 & 30 \\
Heart-Cleveland & 303 & 2 & 6 & 7 & 26 & 1 & 5 & 40 \\
Housing & 506 & 3 & 12 & 1 & 13 & 3 & 2 & 70 \\
Ionosphere & 351 & 2 & 34 & - & 34 & 1 & 10 & 40 \\
Iris & 150 & 3 & 4 & - & 4 & 3 & 5 & 80 \\
Kr-vs-kp & 3196 & 2 & - & 36 & 74 & 1 & 15 & 20 \\
Pima-Diabetes & 768 & 2 & 8 & - & 8 & 1 & 5 & 30 \\
Segmentation & 2310 & 7 & 19 & - & 19 & 7 & 15 & 20 \\
Sonar & 208 & 2 & 60 & - & 60 & 1 & 10 & 60 \\
Vehicle & 846 & 4 & 18 & - & 18 & 4 & 10 & 40 \\
Wine & 178 & 3 & 13 & - & 13 & 3 & 5 & 15 \\
Random21 & 2500 & 2 & 21 & - & 21 & 1 & 1 & 100 \\
Redundant21 & 2500 & 2 & 21 & - & 21 & 1 & 1 & 100 \\
\hline
\end{tabular}

Redundant21. We took the definition of Redundant21 from the paper by Inza et al. [22].

Each numeric feature in the data was linearly normalized to the interval $[-1,1]$. The discrete features and the class labels were encoded with the usual 1-in-C coding if there are $C>2$ values (one of the $C$ outputs is set to 1 and the rest to -1$)$. Binary values were encoded as a single -1 or 1 value.

Instances with missing values in Credit-Australian were deleted. Following the usual practice, the missing values in Pima-Diabetes (denoted with zeroes) were not removed and were treated as if their values were meaningful. Following Lim et al. [23], the classes in Housing were obtained by discretizing the attribute "mean value of owner-occupied homes" as follows: class $=1$ if $\log ($ median value $) \leq 9.84$, class $=2$ if $9.84<\log ($ median value $) \leq 10.075$, and class $=3$ otherwise.

\subsection{Evaluation Method}

To evaluate the generalization accuracy of the pruning methods, we used 5 iterations of 2 -fold crossvalidation $(5 \times 2 \mathrm{cv})$. In each iteration, the data were randomly divided in halves. One half was input to the EAs. The best pruned network found by the EA was tested on the other half of the data. The accuracy results presented in table 2 are the average and standard deviations of the ten tests.

To determine if the differences among the algorithms were statistically significant, we used a combined $\mathrm{F}$ test proposed by Alpaydin [24]. Let $p_{i}^{(j)}$ denote 
Table 2. Mean accuracies found in the $5 \times 2 \mathrm{cv}$ experiments. The best result is in bold and a bullet $(\bullet)$ denotes a result that is significantly different from the best result at a 0.05 level of significance.

\begin{tabular}{l|c|cccc}
\hline Domain & Neural Net & sGA & cGA & ecGA & BOA \\
\hline Breast Cancer & 96.39 & $\mathbf{9 6 . 5 4}$ & 96.13 & 95.84 & 96.42 \\
Cr-Australian & $82.53 \bullet$ & 85.78 & 85.75 & $\mathbf{8 6 . 1 8}$ & 85.84 \\
Cr-German & 70.12 & 70.68 & $\mathbf{7 0 . 9 2}$ & 70.30 & 70.14 \\
Heart-Cleveland & $58.17 \bullet$ & $\mathbf{8 9 . 7 0}$ & 88.05 & 88.78 & 89.37 \\
Housing & $64.62 \bullet$ & $\mathbf{7 5 . 3 6}$ & $67.11 \bullet$ & $64.18 \bullet$ & 76.24 \\
Ionosphere & $\mathbf{8 4 . 7 7}$ & 84.61 & 82.95 & 82.22 & 84.22 \\
Iris & $\mathbf{9 4 . 5 3}$ & 92.93 & $70.13 \bullet$ & $67.73 \bullet$ & 93.60 \\
Kr-vs-kp & $74.30 \bullet$ & 92.56 & 93.53 & 93.81 & $\mathbf{9 3 . 8 5}$ \\
Pima-Diabetes & 73.30 & 74.84 & 75.91 & 76.04 & 75.88 \\
Segmentation & $44.16 \bullet$ & 64.02 & 62.45 & $\mathbf{6 4 . 3 2}$ & 63.66 \\
Sonar & $73.17 \bullet$ & 83.46 & $\mathbf{8 6 . 1 5}$ & 84.90 & 83.55 \\
Vehicle & $69.71 \bullet$ & 78.20 & 76.73 & 76.64 & $\mathbf{7 8 . 6 2}$ \\
Wine & $\mathbf{9 5 . 1 6}$ & 94.15 & $89.88 \bullet$ & $87.41 \bullet$ & 93.48 \\
Random21 & 91.70 & 94.04 & 94.08 & 94.03 & $\mathbf{9 4 . 0 9}$ \\
Redundant21 & 91.75 & 95.77 & $\mathbf{9 5 . 8 2}$ & $\mathbf{9 5 . 8 2}$ & 95.72 \\
\hline
\end{tabular}

the difference in the accuracy rates of two classifiers in fold $j$ of the $i$-th iteration of $5 \times 2 \mathrm{cv}, \bar{p}=\left(p_{i}^{(1)}+p_{i}^{(2)}\right) / 2$ denote the mean, and $s_{i}^{2}=\left(p_{i}^{(1)}-\bar{p}\right)^{2}+\left(p_{i}^{(2)}-\bar{p}\right)^{2}$ the variance, then

$$
f=\frac{\sum_{i=1}^{5} \sum_{j=1}^{2}\left(p_{i}^{(j)}\right)^{2}}{2 \sum_{i=1}^{5} s_{i}^{2}}
$$

is approximately $\mathrm{F}$ distributed with 10 and 5 degrees of freedom, and we rejected the null hypothesis that the two algorithms have the same error rate with 0.95 confidence if $f>4.74$ [24]. The algorithms used the same data partitions and started from identical initial populations.

\section{Experiments}

Table 2 has the average accuracies obtained with each method. The best observed result in the table is highlighted in bold type, and those results that according to the combined $\mathrm{F}$ test are significantly different from the best are marked with a bullet $(\bullet)$.

These results suggest that, in some cases, the pruned networks have significantly higher accuracies than the fully-connected networks. In general, pruning does not seem to have harmful effects on the accuracy, except in two cases (Iris and Wine) where the networks pruned with the cGA and ecGA perform significantly worse than the fully-connected networks. In these experiments the pruned networks were not retrained to "repair the damage" caused by pruning. 
Pruning results in minor accuracy gains over the fully-connected networks, except when the fully-connected nets performed poorly. In those cases, pruning resulted in dramatic improvements. For example, the pruned networks on Heart-Cleveland show improvements of $30 \%$ in accuracy, while in Kr-vs-kp and Segmentation the improvements are $\approx 20 \%$, and in Vehicle the improvements are $\approx 10 \%$.

One reason why pruning might improve the accuracy is because pruning may eliminate the effect of irrelevant or redundant inputs. The experiments with Random 21 and Redundant21 were intended to explore this hypothesis. In Random21, the pruning methods always selected weights corresponding to the nine true inputs, but the algorithms always selected two or three weights corresponding to random inputs. Since the performance does not seem to degrade much, it seems likely that backpropagation had assigned low values to those irrelevant weights. In Redundant21, the pruning methods did not eliminate the redundant features. In fact, the pruned networks retained more than 20 of their 24 weights.

With respect to the size (number of weights) of the final networks, all algorithms had similar results, successfully pruning between 30 and $50 \%$ of the total weights (with the exception of Redundant 21 discussed above).

Table 3 shows that the sGA and the BOA finished in similar number of generations (except for Credit-Australian and Heart-Cleveland), and were in most cases the slowest algorithms. On most data sets, the ecGA finishes faster than the other algorithms. However, the ecGA produced networks with inferior accuracy than the other methods or the fully-connected networks in three cases (Housing, Iris, and Wine). Despite the occasional inferior accuracies, it seems that the ecGA is a good pruning method.

We performed additional experiments retraining the networks after pruning for one, two, and five epochs of backpropagation (results not shown). In most cases, retraining the networks improves the classification accuracy only slightly over pruning without retraining (1-2\%), and there does not appear to be a significant advantage to retrain for more than one epoch. Among the data sets we tested, the largest impact of retraining (using one epoch) was in Housing with an increase of approximately $7 \%$ over pruning without retraing.

Retraining, however, had a large impact on the number of generations until the algorithms terminated. In most cases, retraining for one epoch reduced the generations by approximately $40 \%$. Only in one case (sGA on Random 21 ) the number of generations increased (from 13.6 to 20). Retraining for more than one epoch did not have a noticeable effect on the number of generations. In all instances, retraining increased the total execution time considerably.

The population size of 1024 individuals was chosen because the DEAs require a large population to estimate correctly the parameters of the models of selected individuals. For the simple GAs, it is likely that 1024 individuals is too large a population. In additional experiments, we set the sGA population size to $3 \sqrt{l}$, where $l$ is the size of the chromosomes (number of weights in the network). The only significant difference in accuracy between the sGA with 1024 individuals 
Table 3. Mean generations until termination. The best result is in bold and a bullet $(\bullet)$ denotes a result that is significantly different from the best result at a 0.05 level of significance.

\begin{tabular}{lcccc}
\hline Domain & sGA & cGA & ecGA & BOA \\
\hline Breast Cancer & $9.2 \bullet$ & $\mathbf{6 . 7}$ & 7 & $10.9 \bullet$ \\
Credit-Australian & $\mathbf{1 0}$ & 14 & 14.9 & 14.4 \\
Credit-German & 17.1 & $22.8 \bullet$ & $21.3 \bullet$ & $\mathbf{1 4 . 3}$ \\
Heart-Cleveland & $\mathbf{9 . 8}$ & 10.4 & 10.2 & $15.8 \bullet$ \\
Housing & $19.4 \bullet$ & 7.4 & $\mathbf{7 . 1}$ & $18.6 \bullet$ \\
Ionosphere & 16.8 & 15.7 & $\mathbf{1 5 . 1}$ & 17.8 \\
Iris & $10.1 \bullet$ & $\mathbf{5 . 9}$ & $\mathbf{5 . 9}$ & $10.1 \bullet$ \\
Kr-vs-kp & $37.7 \bullet$ & 28.8 & $\mathbf{2 6}$ & $35.7 \bullet$ \\
Pima-Diabetes & 12.8 & 14.7 & $\mathbf{1 1 . 5}$ & 14.2 \\
Segmentation & $26 \bullet$ & 18.1 & $\mathbf{1 7 . 4}$ & $24.9 \bullet$ \\
Sonar & $\mathbf{1 4 . 5}$ & 20.5 & 19.3 & 16.9 \\
Vehicle & $26.1 \bullet$ & 16.5 & $\mathbf{1 4 . 8}$ & $30.2 \bullet$ \\
Wine & 12.5 & 9.9 & $\mathbf{9 . 4}$ & 11.7 \\
Random21 & 13.6 & $\mathbf{9}$ & 9.1 & $14.8 \bullet$ \\
Redundant21 & $13.7 \bullet$ & $\mathbf{8 . 5}$ & $\mathbf{8 . 5}$ & $16.1 \bullet$ \\
\hline
\end{tabular}

and the smaller population was in Iris $(87.73 \%$ vs. $92.93 \%)$. There were no other significant differences with the best pruning methods. Naturally, the execution time was much shorter with the smaller populations. Therefore, for pruning neural networks, it seems that the best recommendation is a simple GA with small populations.

\section{Conclusions}

This paper presented experiments with four evolutionary algorithms applied to neural network pruning. The experiments considered public-domain and artificial data sets. With these data sets we found that there are few differences in the accuracy of networks pruned by the four EAs, but that the extended compact GA needs fewer generations to finish. However, we also found that, in a few cases, the ecGA results in networks with lower accuracy than those obtained by the other EAs or a fully-connected network.

We also found that retraining the pruned networks seems to improve the classification accuracy only very slightly but incurs in a much higher computational cost. Therefore, it appears that retraining is only recommended in applications where time is not critical.

Additional experiments revealed that a simple GA with a small population can reach results that are not significantly difference from the best pruning methods. Since the smaller populations result in much shorter execution times, the simple GA seems to have an advantage over the other methods.

Future work should explore methods to improve the computational efficiency of the algorithms to deal with much larger data sets. In particular, subsampling 
the training sets and parallelizing the fitness evaluations seem like promising alternatives. Another possible extension of this work is to prune entire units.

\section{Acknowledgments}

I thank Martin Pelikan for providing the graphs in figure 1. UCRL-JC-XXXXXX. This work was performed under the auspices of the U.S. Department of Energy by University of California Lawrence Livermore National Laboratory under contract No. W-7405-Eng-48.

\section{References}

1. Yao, X.: Evolving artificial neural networks. Proceedings of the IEEE 87 (1999) $1423-1447$

2. Castillo, P.A., Arenas, M.G., Castillo-Valdivieso, J.J., Merelo, J.J., Prieto, A., Romero, G.: Artificial neural networks design using evolutionary algorithms. In: Proceedings of the Seventh World Conference on Soft Computing. (2002)

3. Pelikan, M., Goldberg, D.E., Cantú-Paz, E.: BOA: The bayesian optimization algorithm. In Banzhaf, W., Daida, J., Eiben, A.E., Garzon, M.H., Honavar, V., Jakiela, M., Smith, R.E., eds.: Proceedings of the Genetic and Evolutionary Computation Conference 1999: Volume 1, San Francisco, CA, Morgan Kaufmann Publishers (1999) 525-532

4. Etxeberria, R., Larrañaga, P.: Global optimization with Bayesian networks. In: II Symposium on Artificial Intelligence (CIMAF99). (1999) 332-339

5. Mühlenbein, H., Mahnig, T.: FDA-A scalable evolutionary algorithm for the optimization of additively decomposed functions. Evolutionary Computation 7 (1999) 353-376

6. Reed, R.: Pruning algorithms - a survey. IEEE Transactions on Neural Networks 4 (1993) 740-747

7. Whitley, D., Starkweather, T., Bogart, C.: Genetic algorithms and neural networks: Optimizing connections and connectivity. Parallel Computing 14 (1990) 347-361

8. Hancock, P.J.B.: Pruning neural networks by genetic algorithm. In Aleksander, I., Taylor, J., eds.: Proceedings of the 1992 International Conference on Artificial Neural Networks. Volume 2., Amsterdam, Netherlands, Elsevier Science (1992) 991-994

9. LeBaron, B.: An evolutionary bootstrap approach to neural network pruning and generalization. unpublished working paper (1997)

10. Schmidt, M., Stidsen, T.: Using GA to train NN using weight sharing, weight pruning and unit pruning. Technical report, Aarhus University, Computer Science Department, Aarhus, Denmark (1995)

11. Whitley, D., Bogart, C.: The evolution of connectivity: Pruning neural networks using genetic algorithms. Technical Report CS-89-113, Colorado State University, Department of Computer Science, Fort Collins (1989)

12. Thierens, D.: Scalability problems of simple genetic algorithms. Evolutionary Computation 7 (1999) 331-352

13. Pelikan, M., Goldberg, D.E., Lobo, F.: A survey of optimization by building and using probabilistic models. IlliGAL Report No. 99018, University of Illinois at Urbana-Champaign, Illinois Genetic Algorithms Laboratory, Urbana, IL (1999) 
14. Larrañaga, P., Etxeberria, R., Lozano, J.A., Peña, J.M.: Optimization by learning and simulation of Bayesian and Gaussian networks. Tech Report No. EHU-KZAAIK-4/99, University of the Basque Country, Conostia-San Sebastian, Spain (1999)

15. Harik, G.R., Lobo, F.G., Goldberg, D.E.: The compact genetic algorithm. In: Proceedings of the 1998 IEEE International Conference on Evolutionary Computation, Piscataway, NJ, IEEE Service Center (1998) 523-528

16. Baluja, S.: Population-based incremental learning: A method for integrating genetic search based function optimization and competitive learning. Tech. Rep. No. CMU-CS-94-163, Carnegie Mellon University, Pittsburgh, PA (1994)

17. Mühlenbein, H.: The equation for the response to selection and its use for prediction. Evolutionary Computation 5 (1998) 303-346

18. Harik, G.: Linkage learning via probabilistic modeling in the ECGA. IlliGAL Report No. 99010, University of Illinois at Urbana-Champaign, Illinois Genetic Algorithms Laboratory, Urbana, IL (1999)

19. Lobo, F.G., Harik, G.R.: Extended compact genetic algorithm in C++. IlliGAL Report No. 99016, University of Illinois at Urbana-Champaign, Illinois Genetic Algorithms Laboratory, Urbana, IL (1999)

20. Pelikan, M.: A simple implementation of the Bayesian optimization algorithm (BOA) in C++ (version 1.0). IlliGAL Report No. 99011, University of Illinois at Urbana-Champaign, Illinois Genetic Algorithms Laboratory, Urbana, IL (1999)

21. Blake, C., Merz, C.: UCI repository of machine learning databases (1998)

22. Inza, I., Larrañaga, P., Etxeberria, R., Sierra, B.: Feature subset selection by Bayesian networks based on optimization. Artificial Intelligence 123 (1999) 157184

23. Lim, T.J., Loh, W.Y., Shih, Y.S.: A comparison of prediction accuracy, complexity, and training time of thirty-three old and new classification algorithms. Machine Learning 40 (2000) 203-228

24. Alpaydin, E.: Combined $5 \times 2 \mathrm{cv} F$ test for comparing supervised classification algorithms. Neural Computation 11 (1999) 1885-1892 